\title{
La implantación de la estrategia digital en los museos de arte de España: un destino con varias rutas
}

The implementation of the digital strategy in the Spanish art museums: a destination with diverse routes

\section{Adriana Hurtado Jarandilla}

${ }^{\text {a } U n i v e r s i d a d ~ C a r l o s ~ I I I ~ d e ~ M a d r i d . ~ a d r i a n a . h j a r a n d i l l a @ g m a i l . c o m ~}$

\section{Resumen}

La crisis de la COVID-19 nos ha dejado algo claro, y es que la supervivencia de los museos pasa en gran medida por su salto a la esfera digital. Y en este nuevo -y forzado- escenario si hay alguna cuestión que juegue un papel crucial es, indudablemente, la planificación estratégica de la transformación digital. En efecto, el diseño de la estrategia digital resulta imprescindible para que la incorporación de tecnología sea un proyecto sensato y alineado con la misión y los objetivos generales de las instituciones. Así, los museos que ya habian empezado a trabajar en esa línea han visto su labor reconocida y, los que no, se han puesto a ello.

Una vez establecida la estrategia digital, cabe que vayamos un paso más allá y nos preguntemos: ¿quién está a cargo de su correcta implantación? ¿Son los departamentos tradicionales los que tienen que ponerse al frente de esta tarea? ¿O podemos y debemos reivindicar la creación de departamentos ad hoc, centrados en la efectiva transformación digital de las instituciones?

Partiendo de estas preguntas, la finalidad principal de esta comunicación es conocer cómo se implementa la estrategia digital en los museos desde el punto de vista organizativo: a qué equipos o departamentos se les asigna esta tarea y cómo trabajan para realizarla. Lo haremos con un doble enfoque, teórico y práctico. En primer lugar, expondremos cómo la historiografía considera que debe gestionarse la estrategia digital desde la perspectiva del organigrama. A continuación, exploraremos las diferentes formas en que llevan a cabo la implementación digital los museos de arte adscritos a la Red de Museos de España.

Esta comunicación y su doble tratamiento teórico-práctico nos permitirán contraponer el desarrollo teórico con la situación real de la gestión de la estrategia digital. $Y$, con ello, seremos capaces de sentar las bases que permitan a los museos españoles saber en qué punto se encuentran y tomar en el futuro decisiones en este sentido, mejorando con ello la implementación de la estrategia digital. 
Palabras clave: transformación digital, planificación estratégica, estratega digital, organigrama, museología digital

\begin{abstract}
The COVID-19 crisis has provided us with a clear message, which is that the survival of museums mainly involves their leap to the digital sphere. And in this new -and compelled-scenario there's an aspect that plays a fundamental role: the strategic planning of the digital transformation. Indeed, the design of the digital strategy is crucial to allow the incorporation of technology to be a sensible project that is aligned with the mission and the general objectives of institutions. That's why museums who have already started working in this respect have seen their work be recognized and museums who have not done so yet have begun planning it.
\end{abstract}

Once digital strategy is established, we can go one step further and ask: Who is in charge of its implementation? Are the traditional departments the ones who have to take the lead? Or can and should we claim the creation of new digital departments focused on the effective digital transformation of institutions?

Starting with these questions, this communication aims at knowing how digital strategy is implemented in museums from the organizational perspective: Which are the teams or departments in charge of this task and how do they work to achieve the results? We will do it on two levels, theoretical and practical. First, we will present how the historiography consider that digital strategy should be managed from the scope of the organization chart. Then we will explore the different ways in which art museums of the Spanish Museums Network are implementing the digital strategy.

This paper and its double theoretical-practical approach will allow us to compare the theoretical development of this issue with the real performance of the digital strategy management in museums. And thus we will be able to lay the foundations for letting museums know the point they have reached and take future decisions in this respect, improving the implementation of their digital strategies.

Keywords: digital transformation, strategic planning, digital strategist, organizational chart, digital museology 


\section{Introducción}

A estas alturas, no cabe duda de que para gestionar y administrar un museo es necesario planificar estratégicamente lo que se quiere hacer en él pues así lo viene reivindicando la historiografía desde las décadas ochenta y noventa (Hernández, 1994: 103-104; Bravo, 1995 : 177; Moore, 1998: 12 y Moore, 2005: 32). Esto atañe, cómo no, a las iniciativas digitales que se desean emprender, como lo dijera John Perkins en 1994 cuando hizo depender el buen desempeño de los ordenadores en el museo de una correcta planificación (Perkins, 1994: 7). Es pues incuestionable que hoy en día toda incorporación tecnológica debe ir precedida de una estrategia digital que se encuentre alineada con la misión y visión de la institución y con sus objetivos generales (Stein, 2012; Tasich, 2014; Beckett, 2016 y Stein, 2017).

Lo anterior se ha visto reforzado ante la COVID-19 ya que, en la medida en que la pandemia y sus correlativas restricciones provocaron que la supervivencia de los museos pasase necesariamente por su salto a la esfera digital, el papel de la planificación se ha puesto de relieve. Si algo nos ha permitido sacar en claro el confinamiento global es que el diseño de la estrategia digital resulta imprescindible para que la incorporación de tecnología sea un proyecto sensato y alineado con la misión de las instituciones, como resaltaba Suay Aksoy, actual presidenta del Consejo Internacional de Museos (Riaño, 2020). Así, parece claro que los museos que ya habían empezado a trabajar en esa línea han visto su labor reconocida y, los que no, se han puesto a ello. Y una vez establecida la estrategia digital, cabe que vayamos un paso más allá y nos preguntemos: ¿quién está a cargo de su correcta implantación? ¿Son los departamentos tradicionales los que tienen que ponerse al frente de esta tarea? ¿O podemos y debemos reivindicar la creación de departamentos ad hoc -estrategas digitalescentrados en la efectiva transformación digital de las instituciones?

A lo largo de esta comunicación abordaremos las anteriores preguntas y estudiaremos de qué manera los museos de arte de España, una vez planificada su estrategia digital, la han implementado. Para ello, partiremos de una revisión bibliográfica para presentar lo que los expertos recomiendan en torno a la implantación de la estrategia digital. Establecido este marco teórico, descenderemos a un plano práctico y discutiremos quién ejecuta la estrategia digital en los museos españoles, según los resultados obtenidos en una investigación efectuada entre 2017 y 2020.

\section{Objetivos}

Los objetivos que perseguimos con esta comunicación son los siguientes:

1. Conocer cómo, según la historiografía, puede implementarse la estrategia digital en los museos desde el punto de vista organizativo. 
2. Explorar las diferentes maneras en que los museos de arte adscritos a la Red de Museos de España implantan la estrategia digital.

3. Contraponer el desarrollo teórico con el desenvolvimiento práctico -la situación real- de la gestión de la estrategia digital.

4. Sentar las bases que permitan a los museos españoles saber en qué punto se encuentran y tomar en el futuro decisiones que mejoren la implementación de la estrategia digital.

\section{Desarrollo de la investigación}

El fomento de las estrategias digitales ha creado la necesidad de responsabilizar a un trabajador o a un grupo de trabajadores de la correcta puesta en marcha de las estrategias, los proyectos y los planes digitales. Este fenómeno es algo que resulta natural en la medida en que planificar una estrategia es un estadio necesario de la gestión, y un aspecto básico de la dicha gestión es la propia organización de los recursos humanos encargados de materializar los programas y proyectos diseñados por un museo, como expresara Geoffrey Lewis en 1986 (Bravo, 1995: 177). Por ende, entra dentro de la lógica que la aparición de estrategias digitales haya traído consigo la aparición de un estratega digital a cargo de su implantación.

En este sentido, se ha defendido en repetidas ocasiones la importancia de que, pese a que sean uno o varios grupos de trabajadores del museo los responsables directos de la puesta en escena de los proyectos de corte digital, éstos resulten transversales $\mathrm{y}$, en consecuencia, atañan a todos los departamentos ${ }^{1}$. La razón de esto estriba en que la actividad digital tiende a ser holística -raro ámbito de la institución queda fuera de su radio de acción-, de forma que el trabajo en red o interdisciplinar se torna crucial, llegándose a sostener, de hecho, que "para que una estrategia digital que está escrita se lleve a cabo de modo efectivo, tiene que derivar de una estrategia organizativa general" (Tasich, 2014).

Yendo un paso más allá en el razonamiento anterior, recientemente el especialista francés en tecnología para el sector cultural, Omer Pesquer, reivindicaba -al hilo del salto digital de los museos provocado por la COVID-19- que, en tanto el cambio digital afecta a múltiples áreas y funciones del museo, es fundamental que exista un servicio digital transversal específico que evite que la energía de transformación digital se diluya y que vele por que todas las demás áreas ejecutan las estrategias digitales que les atañen (Chapon, 2020).

Por otra parte, al tiempo en que se ha recordado la relevancia de generar sinergias entre todos los departamentos en la planificación, se ha dejado constancia de una práctica cada vez más

\footnotetext{
${ }^{1}$ En la conferencia Museums \& the Web 2016 se afirmó que, de hecho, "el cambio digital es organizativo" y afecta a todos los departamentos (Calvo, 2016). Algo similar sostiene también la especialista española en estrategia digital Conxa Rodà (Rodà (entrevistada), s.f.).
} 
extendida en el día a día de los museos: la contratación de personal ajeno a la propia institución, algo que ya refiriera Francisca Hernández Hernández al asegurar que las funciones más amplias y complejas de estas instituciones han llevado a las mismas "contratar especialistas externos o consultores para tratar determinados problemas" (Hernández, 1994: 78). Estas decisiones de externalización se justifican porque consisten en un ejercicio de racionalización de la gestión (Hernández, 1994: 78) y se han popularizado en mayor medida cuando de lo que se trata es de llevar a cabo la confección y ejecución de planes estratégicos. Al respecto se ha pronunciado Kevin Moore, quien entiende que, aunque la capacidad de planificación late en el interior de cada museo independientemente de cuál sea su tamaño, esto no significa que "los asesores, en algunas circunstancias, no puedan añadir un gran valor a los procesos de planificación estratégica y aumentar notablemente la calidad del plan estratégico resultante" (Moore, 2008: 41-42). Pese a que esta posibilidad de externalizar la planificación parezca algo propio de los museos de mayor tamaño, este autor censura la idea de que únicamente en estos museos sea lícita la contratación de consultores; muy al contrario, sostiene que algunos "relativamente pequeños pueden encontrar los recursos necesarios para contratar asesores en ciertas circunstancias" (Moore, 2008: 41-42).

El recurso a asesores externos al museo para la elaboración de planes gana nuevos adeptos cuando la tarea a planificar no es sino la introducción de nuevas tecnologías. Así por ejemplo, el antes citado John Perkins aseguraba en 1994 que "puede ser útil contratar a un consultor especializado para tratar las importantes cuestiones técnicas" que sirva para dar instrucciones a los proveedores o programadores de la misma (Perkins, 1994: 8-9) ${ }^{2}$.

Así las cosas, quisimos aplicar estos aportes teóricos a la praxis de nuestros museos y efectuar un análisis de los equipos humanos que nos permitiera conocer quién o quiénes están al mando de la ejecución de la estrategia digital. En este proceso, nos interesaba muy particularmente dilucidar si el reparto de tareas en los proyectos digitales es incorporado al propio documento de planificación, encomendándolos a equipos o departamentos específicos $^{3}$. O si, por el contrario, el esfuerzo de diseñar o definir una estrategia digital no va parejo al esfuerzo de delimitar desde el principio quién será el responsable de ésta.

Ante la imposibilidad de llevar a cabo un estudio exhaustivo de todo el ecosistema museístico del país, lo que hicimos fue delimitar los sujetos del estudio a los siguientes nueve museos de arte de la Red de Museos de España: Museo Nacional del Prado, Museo Nacional Centro de Arte Reina Sofía, Museo Nacional Thyssen-Bornemisza, Museo Sorolla, Museo Lázaro Galdiano, Museo Nacional de Escultura, Museo Nacional de Cerámica y Artes Suntuarias

\footnotetext{
2 Por ejemplo, para formar al personal en las herramientas digitales a implantar en la institución (Roca, Llaneza y Carreras, 2009: 115-116).

3 Una idea aconsejada por los expertos, que consideran que una buena estrategia digital debe incluir un reparto claro de las responsabilidades (Beckett, 2016).
} 
“González Martí, Museo Nacional de Artes Decorativas y Museo Cerralbo. El motivo por el que se optó por restringir la investigación a estas instituciones se encuentra en que la misma se desarrolló en el marco de la tesis doctoral que realicé en la Universidad Carlos III de Madrid entre los años 2017 y 2020, en la que restringí la muestra a los once museos de arte de dicha red ${ }^{4}$ para poder efectuar un análisis más profundo. Para esta comunicación, se excluyeron a los museos del Greco y del Romanticismo porque tales instituciones no tienen documentos de planificación propiamente dichos y, por consiguiente, no podemos predicar que los mismos cuenten con una estrategia digital a implantar, tal y como comentamos en una investigación previa (Hurtado, 2019: 32).

Los métodos adoptados para realizar esta investigación consistieron en la revisión crítica de los documentos de planificación estratégica de los museos seleccionados y la consulta de los organigramas de las instituciones para entender el reparto de las tareas digitales. Con esta finalidad visitamos los sitios webs, que en algunos casos nos permitieron estudiar tanto los planes estratégicos como sus estructuras organizativas; en otros, en cambio, esta consulta fue insuficiente porque no publican tales documentos o porque no aportan información sobre sus organigramas. En esos supuestos, fue de rigor acudir a los profesionales que trabajan en estas instituciones para que nos facilitaran la información faltante ${ }^{5}$.

\section{Resultados}

Sobre la contratación de empresas externas, cabe decir que, en términos generales, ninguno de los museos estudiados encomienda la implantación de la estrategia digital a expertos ajenos a la institución. Todo ello sin perjuicio de que, en algunos casos, en los planes sí se prevea el recurso a empresas para externalizar determinados servicios, o acuerdos de colaboración con empresas para realizar proyectos digitales específicos (Ministerio de Cultura, 2011: 3-4, 21; ALS y Museo Thyssen-Bornemisza, 2013: 408, 537, 688; Fundación Lázaro Galdiano, 2015: 5, 11; Ministerio de Educación, Cultura y Deporte, 2017: 51-52; Fundación Lázaro Galdiano, 2018: 6, 12, y Museo Nacional Thyssen-Bornemisza, 2018: 17, 19 44). Además, en el Museo Nacional Thyssen-Bornemisza, si bien no se externaliza la implementación de la estrategia digital, sí que se recurrió a expertos ajenos al museo para llevar a cabo el diseño de estrategias digitales. En efecto, esta institución encargó la

\footnotetext{
${ }^{4}$ Los museos enunciados y, además, el Museo del Greco y el Museo del Romanticismo.

${ }^{5} \mathrm{Al}$ respecto de la falta de publicación de planes estratégicos, esto sucedió en el Museo Cerralbo, en el Museo Nacional de Escultura y en el Museo Nacional de Cerámica y Artes Suntuarias “González Martí". En el Thyssen-Bornemisza también nos dieron acceso a su plan para el período 2016-2018 porque no estaba publicado en su momento; sin embargo y afortunadamente, el actualmente vigente está publicado en la web. En cuanto a los organigramas, el Cerralbo fue el único que no publica esta información y, por ello, tuvimos que acudir a sus profesionales, que nos facilitaron a esta información.
} 
elaboración de su plan estratégico para el período 2013-2016 a la empresa Advanced Leisure Services (ASL y Museo Thyssen-Bornemisza, 2013).

A continuación, quisimos saber si en los planes estratégicos se asignaban los proyectos digitales a equipos o departamentos concretos. Esta práctica sólo la pudimos observar en el Museo del Prado, el Museo de Escultura, el Museo de Cerámica y el Thyssen-Bornemisza.

En cuanto al Prado, a propósito de la línea de actuación "Prado Digital" se diseña un subprograma que lleva por nombre "Optimización de Recursos y Transversalidad" que busca “coordinar la adaptación de las diferentes áreas del Museo al entorno digital para lograr un uso más eficiente de los recursos" (Ministerio de Educación, Cultura y Deporte, 2017: 57). Para ello, se pretende la creación de un sistema de documentación de la actividad que permita generar un entorno de colaboración y una herramienta de trabajo, al mismo tiempo que se desea elaborar y difundir "proyectos colaborativos desde un enfoque digital, en los que participan las diferentes áreas promoviendo la sinergia entre ellas" (Ministerio de Educación, Cultura y Deporte, 2017: 57). En consonancia, el museo establece la "creación de grupos de trabajo transversales entre diferentes áreas $[\ldots]$ para la elaboración de nuevas propuestas de cara a la difusión y programación de las actividades y colecciones del Museo desde el enfoque digital" (Ministerio de Educación, Cultura y Deporte, 2017: 57) por lo que, si bien constataremos a continuación la existencia de un concreto Área de Desarrollo Digital, la institución no pretende que los asuntos digitales sean de implicación exclusiva de este departamento sino que se potencie el diálogo y se tiendan puentes entre todos los trabajadores.

En el Thyssen-Bornemisza, cuando se elaboró el plan 2013-2018 (en 2012) las cuestiones digitales eran responsabilidad de diversos equipos y de la intersección entre ellos. Así, de la atención del visitante antes de la visita y después en redes sociales, se encargaba el equipo de informática (ASL y Museo Thyssen-Bornemisza, 2013: 37), y de la difusión en redes sociales era responsable el Departamento de Comunicación en colaboración con el de Informática (ASL y Museo Thyssen-Bornemisza, 2013: 40). De hecho, en la definición de los programas que contiene el plan se hace una descripción pormenorizada de todos los planes de acción, predicando en el de "Presencia on-line" la involucración necesaria del Director gerente y de los Departamentos de Informática, Educación, Comunicación y Tiendapublicaciones. El liderazgo recae en uno o en otro según sea la acción. En los programas sobre desarrollo de la estrategia y rediseño de la web, es líder el Director gerente y colaboran Informática, Educación y Comunicación (ASL y Museo Thyssen-Bornemisza, 2013: 686687); el plan de medios sociales es tarea liderada por Comunicación con apoyo de Informática (ASL y Museo Thyssen-Bornemisza, 2013: 688), y la acción para la reutilizacióncomercialización de contenidos digitales corre a cargo del Director gerente con ayuda de Informática, Comunicación, Tienda-Publicaciones y Educación (ASL y Museo ThyssenBornemisza, 2013: 689). 
El nuevo plan 2019-2023, en cambio, no hace una distribución tan clara de los proyectos a las diversas áreas de la institución. Además (y por lo que refiere particularmente a la transformación digital), aunque aborda de una forma extendida el área de Tecnología, nada dice sobre las funciones específicas que éste tiene encomendadas, más allá de toda referencia a la tecnología como elemento de cohesión en el Museo (Museo Nacional ThyssenBornemisza, 2018: 37-38). En cualquier caso, se da por hecho, por la propia redacción del plan, que esta área será la encargada de coordinar todo proyecto digital con independencia de que refiera a otro departamento o área distinto al tecnológico.

El Museo Nacional de Escultura, a su vez, distribuye los objetivos específicos del plan según áreas, de manera que cada una de ellas es responsable del cumplimiento de determinadas metas. Y, por ello, la estrategia digital no queda constreñida a un departamento en concreto; por el contrario, es competencia de todos (Museo Nacional de Escultura, s.f.: 6-8):

- Conservación Preventiva y Restauración es responsable de la mejora en los equipos de restauración y fotográficos.

- $\mathrm{Al}$ área Biblioteca le ha sido encomendado todo lo relacionado con la digitalización y la catalogación de los fondos.

- Colecciones se hace cargo de la edición de la publicación digital.

- Los departamentos Difusión y Colección son los encargados de trabajar en las nuevas producciones para los soportes multimedia.

Por último, el Museo Nacional de Cerámica, aunque de un modo sucinto, indica que los proyectos digitales son fundamentalmente tarea del departamento de Difusión $\mathrm{y}$, en ocasiones, de especialistas externos (Ministerio de Cultura, 2011: 21).

El siguiente paso consistió en la consulta de los organigramas de las instituciones. En esta fase, nos percatamos de que existen diferentes prácticas. En primer lugar, encontramos el caso de los museos en cuyos organigramas se prevén puestos específicos de desarrollo digital:

- El Prado cuenta con un Área de Desarrollo Digital que engloba el Servicio de Informática y el Servicio Web;

- En el MNCARS existe un Responsable de Programas Virtuales (perteneciente al departamento de Actividades Públicas) y un Jefe de Informática (adscrito a la Subdirección General Gerencia) ${ }^{6}$;

- El Thyssen-Bornemisza dispone un departamento de Tecnología dependiente de la Dirección - gerencia, y

\footnotetext{
${ }^{6}$ Aunque prevé unos responsables específicos de la estrategia digital, este museo, en un reportaje reciente, celebraba que la incorporación de la tecnología en el museo potencia precisamente la colaboración entre departamentos; por ello, también en esta institución podemos hablar de la transversalidad de la implementación de la estrategia digital (Hermoso, 2020).
} 
- En el Lázaro Galdiano, dependiente del Gerente adjunto, encontramos un Responsable de Página Web y Nuevos Medios.

A continuación, encontramos el caso del Museo Nacional de Artes Decorativas, donde se proclama la transversalidad de un modo genérico. Y es que, a pesar de no existir un departamento o área dedicada a asuntos digitales, en la propia página web de la institución se afirma que se adopta una "metodología de trabajo transversal por objetivos y proyectos, concebidos y ejecutados por equipos de trabajo mixtos, formados por los técnicos de los distintos departamentos". Por consiguiente, puede predicarse respecto del MNAD que sus proyectos digitales serán materia de trabajo de varios departamentos simultáneamente.

Por último, en el Museo de Escultura, el Museo de Cerámica, el Museo Cerralbo y el Museo Sorolla, también puede hablarse de cierta transversalidad de lo digital. En efecto, como cada área es responsable de ejecutar los proyectos que les atañan en virtud de las competencias o tareas que les hayan sido asignadas, se entiende que lo digital les llegará según sea o no tangencial a sus funciones.

\section{Conclusiones}

En primer lugar hemos de indicar que, pese a que los expertos hablen de la pertinencia de que los documentos de planificación prevean un reparto claro de la responsabilidad de los proyectos digitales, ésta no es la tónica habitual entre los museos analizados (solamente cuatro de los nueve museos sí lo hacen). Por ello, es necesario incidir en esta cuestión: no hay planificación digital efectiva -o los ejercicios de planificación pierden fuelle- si se olvida que la asignación de las tareas y de los proyectos de naturaleza digital es parte de la misma.

Por otra parte, la historiografía hace hincapié en la transversalidad de la implementación de la estrategia digital como consecuencia del carácter holístico de la transformación digital, algo que afortunadamente tiene un reflejo en la praxis porque en todos los museos investigados se proclama la transversalidad de las estrategias digitales de una u otra manera.

En cuanto a la creación de departamentos específicos a cargo de la estrategia digital, esto que permite una mayor vigilancia y un control y seguimiento de los resultados de los proyectos digitales- es minoritario (tan sólo en el Prado, el Thyssen-Bornemisza, el Reina Sofía y el Lázaro Galdiano existen este tipo de departamentos).

Para terminar, respecto a la externalización de la transformación digital que algunos expertos defienden como mecanismo para agilizar la gestión, en términos generales vemos cómo los trabajadores de los museos siguen siendo los responsables de la ejecución de las estrategias digitales. Sin embargo, sí que algunos museos (el de Cerámica, el Lázaro Galdiano, el Prado 
y el Thyssen-Bornemisza) prevén en sus documentos de planificación la colaboración con empresas para que éstas desarrollen puntualmente proyectos digitales. Ahora bien, el hecho de que no aparezca planificado este recurso a terceras entidades no significa que los museos, de facto, no lleven a cabo tales externalizaciones; al contrario, es una práctica bastante extendida en el panorama museístico. En este sentido, consideramos que, en la medida en que es inevitable que así suceda (porque las empresas permiten encarar la falta de recursos en las instituciones para emprender proyectos digitales), sería conveniente que la colaboración público-privada se previera en los documentos estratégicos para, de esta forma, asegurar que los proyectos conjuntos cumplen unos criterios éticos y que la participación del sector privado en el museo es siempre en beneficio de la institución, de su misión y de la sociedad a la que sirve.

\section{Referencias}

\section{Libro}

ALS Y MUSEO THYSSEN-BORNEMISZA (2013). Plan Estratégico del Museo Thyssen-Bornemisza 2013- 2018. Madrid.

FUNDACIÓN LÁZARO GALDIANO (2015). Plan Estratégico 2016-2018. Madrid. $<$ http://www.flg.es/documentos/transparencia/plan-estrategico-2016-2018-museo-lazarogaldiano.pdf $>$ [Consulta: 20 de febrero de 2021]

FUNDACIÓN LÁZARO GALDIANO (2018). Plan Estratégico 2019-2022. Madrid $<$ http://www.flg.es/documentos/transparencia/plan-estrategico-2019-2022-museo-lazarogaldiano.pdf $>$ [Consulta: 20 de febrero de 2021]

HERNÁNDEZ HERNÁNDEZ, F. (1994). Manual de museología. Madrid: Síntesis.

MINISTERIO DE CULTURA (2011). Plan Director del Museo Nacional de Cerámica y Artes Suntuarias "González Marti". Valencia.

MINISTERIO DE EDUCACIÓN, CULTURA Y DEPORTE (2011). Visión, finalidad, objetivos, politicas y programas del Museo Nacional de Artes Decorativas 2011-2015. Madrid. $<$ http://www.culturaydeporte.gob.es/mnartesdecorativas/dam/jcr:ec985b16-868b-4ae9-9ea68414daf35614/plan-director.pdf $>$ [Consulta: 12 de febrero de 2021]

MINISTERIO DE EDUCACIÓN, CULTURA Y DEPORTE (2017). Museo Nacional del Prado: Plan de Actuación 2017-2020. Madrid. <https://content1.cdnprado.net/doclinks/pdf/museo/planactuacion/plan_actuacion_2017.pdf> [Consulta: 12 de febrero de 2021]

MOORE, K. (ed.) (1998). La gestión del museo. Asturias: Trea.

MUSEO CERRALBO (s.f.). Plan Director. Madrid.

MUSEO NACIONAL DE ESCULTURA (s.f.). Plan Director. Valladolid.

MUSEO NACIONAL THYSSEN-BORNEMISZA (2018). Plan Estratégico 2019-2023 de la Fundación Colección Thyssen-Bornemisza F.D.P. Madrid. 
$<$ https://imagenes.museothyssen.org/sites/default/files/document/201908/Plan_estrategico_2019_2023.pdf> [Consulta: 12 de febrero de 2021]

MUSEO SOROLLA (s.f.). Plan Director 2012-2015. Madrid. $<$ http://www.culturaydeporte.gob.es/msorolla/dam/jcr:860dec9d-b9dc-4e34-82974cf62f67e439/plan-director-maquetado.pdf $>$ [Consulta: 12 de febrero de 2021]

\section{Capítulo de un libro}

MOORE, K. (2008). "Tengo un sueño. Planificación estratégica como inspiración para los museos” en VV.AA. Actas de las I Jornadas de Formación Museológica. Museos y planificación: estrategias de futuro. Madrid: Ministerio de Cultura. $<\mathrm{http} / / / \mathrm{www}$.ibermuseos.org/recursos/documentos/actasde-las-primeras-jornadas-de-formacion-museologica-museos-y-planificacion-estrategias-defuturo/> [Consulta: 12 de febrero de 2021]

ROCA, B., LLANEZA, M., CARRERAS MONFORT, C. (2009). "Capítulo IV. Operadores culturales" en Carreras Monfort, C. (ed.). Evaluación TIC en el patrimonio cultural: metodologías y estudios de caso. Barcelona: Editorial UOC.

STAIN, R. (2012). "Blow Up Your Digital Strategy: Changing the Conversation about Museums and Technology" en Proctor, N. y Cherry, R. (eds.). Museums and the Web 2012. Maryland: MD: Museums and the Web. $<$ http://www.museumsandtheweb.com/mw2012/papers/blow_up_your_digital_strategy_changing _the_c_1> [Consulta: 13 de febrero de 2021]

\section{Artículo de una revista o periódico}

BRAVO JUEGA, I. (1995). "La organización y gestión de museos" en Boletín de la ANABAD, vol. 45, n. 1, p. 177-194.

CHAPON, B. (2020). “« Nuit des Musées » : « Etre seul pendant sa visite virtuelle, c’est dépriment », explique Omer Pesquer" en 20 minutes, 14 de noviembre. <https://www.20minutes.fr/artsstars/culture/2907999-20201114-nuit-musees-etre-seul-pendant-visite-virtuelle-deprimantexplique-omer-pesquer $>$ [Consulta: 13 de febrero de 2021]

HERMOSO, B. (2020). "El Reina Sofía afronta su encrucijada” en El País Semanal, 20 de septiembre. $<$ https://elpais.com/elpais/2020/09/22/eps/1600767137_387014.html> [Consulta: 20 de febrero de 2021]

HURTADO JARANDILLA, A. (2019). "La planificación estratégica de la transformación digital. El caso de los museos españoles" en RdM. Revista de Museología: Publicación cientifica al servicio de la comunidad museológica, n. 74, pp. 25-38.

RIAÑO, P. H. (2020). "Suay Aksoy: «Muchos museos no van a volver a abrir»" en El País, 16 de mayo. <https://elpais.com/cultura/2020-05-15/suay-aksoy-muchos-museos-no-van-a-volver-aabrir.html $>$ [Consulta: 13 de febrero de 2021]

MOORE, K. (2005). "La planificación estratégica en los museos" en Museos.es: Revista de la Subdirección General de Museos Estatales, n. 1, p. 32-47. $<$ http://www.culturaydeporte.gob.es/dam/jcr:249a5c73-97be-446f-bd97-635136c3b037/s22planificacion.pdf> [Consulta: 13 de febrero de 2021]

PERKINS, J. (1994). "Partir de cero: la introducción de computadoras en los museos" en Museum Internacional, vol. XLVI, n. 1, p. 7-11. 


\section{Página web}

ADVANCED LEISURE SERVICES. $A L S .<$ http://www.adleisure.com/> [Consulta: 13 de febrero de 2021]

MUSEO CERRALBO. MUSEO CERRALBO - Museo Cerraldo | Ministerio de Cultura y Deporte. $<$ https://www.culturaydeporte.gob.es/mcerralbo/> [Consulta: 11 de febrero de 2021]

MUSEO DEL GRECO. El Museo del Greco-MUSEO DEL GRECO | Ministerio de Cultura y Deporte. $<$ http://www.culturaydeporte.gob.es/mgreco/> [Consulta: 11 de febrero de 2021]

MUSEO LÁZARO GALDIANO. Museo Lázaro Galdiano. Un Museo para el Coleccionismo en Madrid. <http://www.flg.es/> [Consulta: 11 de febrero de 2021]

MUSEO NACIONAL CENTRO DE ARTE REINA SOFÍA. Museo Nacional Centro de Arte Reina Sofia. $<$ http://www.museoreinasofia.es/> [Consulta: 11 de febrero de 2021]

MUSEO NACIONAL DE ARTES DECORATIVAS. Portada-Museo Nacional de Artes Decorativas | Ministerio de Cultura y Deporte. <http://www.culturaydeporte.gob.es/mnartesdecorativas/> [Consulta: 11 de febrero de 2021]

MUSEO NACIONAL DE CERÁMICA Y ARTES SUNTUARIAS “GONZÁLEZ MARTÍ”. Inicio Museo Nacional de Cerámica y Artes Suntuarias "González Marti”" | Ministerio de Cultura y Deporte. <http://www.culturaydeporte.gob.es/mnceramica/> [Consulta: 11 de febrero de 20201]

MUSEO NACIONAL DE ESCULTURA. Portada - Museo Nacional de Escultura | Ministerio de Cultura y Deporte. <http://www.culturaydeporte.gob.es/mnescultura/> [Consulta: 11 de febrero de 2021]

MUSEO NACIONAL DEL PRADO. Museo Nacional del Prado. <https://www.museodelprado.es/> [Consulta: 11 de febrero de 2021]

MUSEO NACIONAL DEL ROMANTICISMO. Inicio - Museo del Romanticismo | Ministerio de Cultura y Deporte. <http://www.culturaydeporte.gob.es/mromanticismo/inicio.html $>$ [Consulta: 11 de febrero de 2021]

MUSEO NACIONAL THYSSEN-BORNEMISZA. Museo Nacional Thyssen-Bormemisza Madrid. $<$ https://www.museothyssen.org/> [Consulta : 11 de febrero de 2021]

MUSEO SOROLlA. Portada - Museo Sorolla | Ministerio de Cultura y Deporte. $<$ http://www.culturaydeporte.gob.es/msorolla/> [Consulta: 11 de febrero de 2021]

\section{Blogs}

BECKETT, C. (2016). "La transformación digital en las organizaciones culturales" en CCCBLAB Investigación e Innovación en Cultura, 31 de mayo. <http://lab.cccb.org/es/la-transformaciondigital-en-las-organizaciones-culturales/> [Consulta: 13 de febrero de 2021]

CALVO, L. (2016). "Museums \& the Web: 20 años de debate sobre la transformación digital" en CCCBLAB Investigación e Innovación en Cultura, 10 de mayo. $<\mathrm{http}: / / \mathrm{lab} . c c c b . o r g / e s / m u s e u m s-$ and-the-web-20-anos-de-debate-sobre-la-transformacion-digital/> [Consulta: 12 de febrero de 2021]

RODÀ, C. (entrevistada) (s.f.). "Conxa Rodà: La innovación digital requiere actitud y aptitud” en Museos e Institutos Nacionales, s.f. <https://museos.cultura.gob.ar/noticia/conxa-roda-lainnovacion-digital-requiere-actitud-y-aptitud/> [Consulta : 12 de febrero de 2021] 
STEIN, R. (2017). "Museums and Digital Strategy Today" en American Alliance of Museums, 10 de julio. < https://www.aam-us.org/2017/07/10/museums-and-digital-strategy-today/> [Consulta: 13 de febrero de 2021]

TASICH, T. (2014). "Estrategias de transformación de los museos en la era digital" en CCCBLAB Investigación e Innovación en Cultura, 26 de febrero. <http://lab.cccb.org/es/estrategias-detransformacion-de-los-museos-en-la-era-digital/> [Consulta: 12 de febrero de 2021] 